\title{
Mixtures of Gaussians on Tensor Fields for DT-MRI Segmentation
}

\author{
Rodrigo de Luis-García and Carlos Alberola-López \\ ETSI Telecomunicación, University of Valladolid, Valladolid, Spain \\ $\{$ rodlui, caralb\}@tel.uva.es
}

\begin{abstract}
In this paper, an original approach for the segmentation of tensor fields is proposed. Based on the modeling of the data by means of Gaussian mixtures directly in the tensor domain, this technique presents a wide range of applications in medical image processing, particularly for Diffusion Tensor Magnetic Resonance Imaging (DT-MRI). The performance of the segmentation method proposed is shown through the segmentation of the corpus callosum from a dataset of 32 DT-MRI volumes. Comparison with a recent and related segmentation approach is favorable to our method, showing its capability for the automatic extraction of anatomical structures in the white matter.
\end{abstract}

\section{Introduction}

DT-MRI is a medical imaging modality which provides, at each voxel, a symmetric second order tensor that describes the diffusion of water molecules 1 . The diffusion tensor can be thought of as the covariance matrix of a zero-mean trivariate Gaussian distribution that models the diffusion, and is represented by a symmetric and positive definite (SPD) $3 \times 3$ matrix. Brain imaging is the most common application of diffusion MRI, as it can be employed for the visualization of the fibre tracts in the white matter of the brain.

DT-MRI has proved to be particularly relevant in a wide range of neurological clinical pathologies, such as ischemia, multiple sclerosis or schizophrenia, among others. The interested reader is referred to [23] for a comprehensive introduction to the applications of DT-MRI to brain diseases.

The segmentation of anatomical structures from DT-MRI data is a relatively new topic in medical image processing. In the last years, several authors have addressed the issue of segmentation of white matter internal structures from DTMRI data, such as the corpus callosum or the corona radiata 4/567/8 91011 .

Initially, most of the methods intended for the segmentation of tensor fields were based on scalar or vector values extracted from the tensors [4. Later, segmentation methods have been proposed working directly in the tensor domain, using tensor dissimilarity measures. Feddern et al. first proposed a level set segmentation method that directly worked on tensor data [5]. In [6] a modified $k$-means algorithm was proposed by Wiegell et al. to segment the thalamic nuclei from DT-MRI, using a combination of the Mahalanobis voxel distance and the Frobenius tensor distance.

N. Ayache, S. Ourselin, A. Maeder (Eds.): MICCAI 2007, Part I, LNCS 4791, pp. 319 326, 2007.

(C) Springer-Verlag Berlin Heidelberg 2007 
In [12, Wang and Vemuri proposed the use of a region-based active contour model for the segmentation of tensor fields, using the Frobenius norm of the difference of tensors as a tensor dissimilarity measure.

Jonasson et al., in [11, faced the segmentation of DT-MRI data from a perspective somehow inspired on a fibre tracking approach. In a level set framework, the speed propagation of the front is proportional to the similarity between the tensors lying on the front and its neighbors, measured in terms of the normalized tensor scalar product.

Wang and Vemuri introduced in [7] a new tensor dissimilarity measure, the Kullback-Leibler (KL) divergence. Starting from the KL divergence, a level set is evolved minimizing an energy functional derived from the Active Contours Without Edges model [13. This segmentation approach was successfully tested on real DT-MRI data. Its main drawback is, coherently with the Chan \& Vese model on which it is grounded, its limitation to a piecewise constant model.

In [8], Lenglet et al. presented a segmentation approach that proposes the minimization of an energy functional based on the Gaussian modeling of the KL distances from the tensor at each voxel to the mean tensor over each region, thus improving the segmentation with the Chan \& Vese model. A new tensor dissimilarity measure, the geodesic distance, was also introduced and applied in this segmentation approach.

Later, Lenglet et al. proposed a definition of Gaussian distributions between diffusion tensors 1410, that are incorporated into the probabilistic setting of the Geodesic Active Regions (GAR) model [15. This segmentation method has shown to be capable of successfully segmenting internal white matter anatomical structures such as the corpus callosum. However, its performance is limited by the Gaussian modeling of the data; problems can be encountered if the complexity of the data is high enough so as to render the model too simplistic, a fact that affects the robustness and accuracy of the segmentation.

In this paper, a novel segmentation method for tensor-valued data is presented based on the definition of mixtures of Gaussians (MoG) over tensor fields. The model introduced here is, consequently, a generalization of the former proposals, and it is aimed at providing more flexibility in the segmentation and the capability to deal with more complex distributions in the data. The segmentation is performed in a region-oriented level set framework, following the GAR model.

In order to illustrate the performance of the proposed segmentation method, the corpus callosum has been segmented out of a set of 32 DT-MRI volumes. The segmentation of this anatomical structure has been addressed in many of the tensor field segmentation approaches in the literature 48191011, and is therefore a good choice for benchmarking. However, the performance of the segmentation approaches in the literature has been customarily validated on a single or few volumes, and a study on a larger dataset has not been performed before.

The remainder of this paper is organized as follows: next section presents the proposed segmentation method, from the definition of MoG on tensors to the derivation of the level set evolution equation. In Section 3, experimental work 
is presented and discussed in order to validate the method proposed. Finally, a brief summary is presented.

\section{Segmentation Method}

\subsection{Gaussian Mixtures on Tensors}

In the work by Lenglet et al. 1410, a Gaussian distribution between tensors belonging to the manifold $S^{+}(3, \mathbb{R})$ of the $3 \times 3$ real, SPD matrices was defined with the following probability density function $(\mathrm{PDF})$ :

$$
p\left(\mathbf{T}_{i} \mid \overline{\mathbf{T}}, \boldsymbol{\Lambda}\right)=\frac{1}{\sqrt{(2 \pi)^{6}|\boldsymbol{\Lambda}|}} \exp \left(-\frac{\boldsymbol{\varphi}\left(\boldsymbol{\beta}_{i}\right)^{T} \boldsymbol{\Lambda}^{-1} \boldsymbol{\varphi}\left(\boldsymbol{\beta}_{i}\right)}{2}\right)
$$

The following elements need to be specified to complete the description of the Gaussian distribution:

$-\mathbf{T}_{i}$ is the $3 \times 3$ tensor located at voxel $i$ in $\Omega$.

- $\overline{\mathbf{T}}$ is the empirical mean tensor over a set of $N$ diffusion tensors.

$-\boldsymbol{\Lambda}$ is the associated covariance matrix, whose size is $6 \times 6$ for a $3 \times 3$ tensor (that is, the number of free components).

- The symmetric matrix $\boldsymbol{\beta}_{i}$ is defined, for a given metric $\mathcal{D}_{x}$, by $\boldsymbol{\beta}_{i}=-\nabla_{\mathbf{T}_{\mathbf{i}}} \mathcal{D}_{x}$ $\left(\mathbf{T}_{i}, \overline{\mathbf{T}}\right)$.

- The map $\varphi: S^{+}(3, \mathbb{R}) \mapsto \mathbb{R}^{6}$ associates to each matrix $\boldsymbol{\beta}_{i}$ its 6 free components.

The definition of the Gaussian distribution is general in terms of the metric employed. In their work, Lenglet et al. considered three different choices: Euclidean metric, KL divergence and geodesic distance. As empirical evidence favours the geodesic distance [1410, it will be employed in this work.

Starting from the definition of Gaussian PDF over tensor fields defined before, we now define a new PDF composed of a mixture of Gaussians. For a mixture of $K$ Gaussians, the PDF for a tensor $\mathbf{T}_{i}$ will be:

$$
p\left(\mathbf{T}_{i} \mid \boldsymbol{\Theta}\right)=\sum_{k=1}^{K} \alpha_{k} \frac{1}{\sqrt{(2 \pi)^{d}\left|\boldsymbol{\Lambda}_{k}\right|}} \exp \left(-\frac{\boldsymbol{\varphi}\left(\boldsymbol{\beta}_{i, k}\right)^{T} \boldsymbol{\Lambda}_{k}^{-1} \boldsymbol{\varphi}\left(\boldsymbol{\beta}_{i, k}\right)}{2}\right)
$$

where we denote by $\Theta$ the set of parameters: $\alpha_{k}, k=1, \ldots, K$ are the mixing probabilities of the different components of the mixture, and each Gaussian distribution is characterized by its mean tensor $\overline{\mathbf{T}}_{k}$ and its covariance matrix $\boldsymbol{\Lambda}_{k}$.

In order to estimate the parameter vector $\boldsymbol{\Theta}$, a Maximum Likelihood (ML) approach by means of the EM algorithm will be followed, as it is customary for Gaussian mixtures. Hereafter, and in order to simplify the notation, we will express the dependencies of $\boldsymbol{\varphi}_{i}$ simply as $\boldsymbol{\varphi}_{i}\left(\overline{\mathbf{T}}_{k}\right)$ (note that $\boldsymbol{\varphi}_{i}=\boldsymbol{\varphi}\left(\boldsymbol{\beta}_{i}\right)$ and $\boldsymbol{\beta}_{i}=\boldsymbol{\beta}_{i}\left(\overline{\mathbf{T}}_{k}\right)$. The log-likelihood we seek to maximize will be given by

$$
\log (\mathcal{L})=\sum_{i=1}^{N} \log \left(p\left(\mathbf{T}_{i} \mid \boldsymbol{\Theta}\right)\right)=\sum_{i=1}^{N} \log \left(\sum_{k=1}^{K} \alpha_{k} g_{k}\left(\mathbf{T}_{i} \mid \boldsymbol{\Theta}_{k}\right)\right)
$$


where we have denoted by $g_{k}\left(\mathbf{T}_{i} \mid \boldsymbol{\Theta}_{k}\right)$ the PDF of each of the components of the mixture, with parameters $\boldsymbol{\Theta}_{k}=\left\{\alpha_{k}, \overline{\mathbf{T}}_{k}, \boldsymbol{\Lambda}_{k}\right\}$. We first derive the log-likelihood with respect to the mean tensor $\overline{\mathbf{T}}_{k}$ :

$$
\begin{aligned}
& \frac{\partial}{\partial \overline{\mathbf{T}}_{k}} \log (\mathcal{L})=\sum_{i=1}^{N} \frac{1}{\sum_{k=1}^{K} \alpha_{k} g_{k}\left(\mathbf{T}_{i} \mid \boldsymbol{\Theta}_{k}\right)} \frac{\partial}{\partial \overline{\mathbf{T}}_{k}}\left(\sum_{k=1}^{K} \alpha_{k} g_{k}\left(\mathbf{T}_{i} \mid \boldsymbol{\Theta}_{k}\right)\right) \\
= & \sum_{i=1}^{N} \frac{1}{\sum_{k=1}^{K} \alpha_{k} g_{k}\left(\mathbf{T}_{i} \mid \boldsymbol{\Theta}_{k}\right)} \alpha_{k} g_{k}\left(\mathbf{T}_{i} \mid \boldsymbol{\Theta}_{k}\right) \frac{\partial}{\partial \overline{\mathbf{T}}_{k}}\left[-\frac{1}{2}\left(\boldsymbol{\varphi}_{i}\left(\overline{\mathbf{T}}_{k}\right)\right)^{T} \boldsymbol{\Lambda}_{k}^{-1}\left(\boldsymbol{\varphi}_{i}\left(\overline{\mathbf{T}}_{k}\right)\right)\right]
\end{aligned}
$$

In order to provide a more compact expression, we can reformulate the Gaussian distributions on tensors as distributions on vectors, where tensor $\mathbf{T}_{i}$ is represented by its free components, $\mathbf{t}_{i}$. Using this representation, the derivative we need to compute can be rewritten:

$$
\begin{aligned}
\frac{\partial}{\partial \overline{\mathbf{t}}_{k}}\left[\left(\boldsymbol{\varphi}_{i}\left(\overline{\mathbf{t}}_{k}\right)\right)^{T} \boldsymbol{\Lambda}^{-1}\left(\boldsymbol{\varphi}_{i}\left(\overline{\mathbf{t}}_{k}\right)\right)\right] & =\frac{\partial}{\partial \boldsymbol{\varphi}_{i}}\left[\left(\boldsymbol{\varphi}_{i}\left(\overline{\mathbf{t}}_{k}\right)\right)^{T} \boldsymbol{\Lambda}^{-1}\left(\boldsymbol{\varphi}_{i}\left(\overline{\mathbf{t}}_{k}\right)\right)\right] \frac{\partial \boldsymbol{\varphi}_{i}}{\partial \overline{\mathbf{t}}_{k}} \\
& =\left(\boldsymbol{\varphi}_{i}\left(\overline{\mathbf{t}}_{k}\right)\right)^{T} \boldsymbol{\Lambda}^{-1} \frac{\partial \boldsymbol{\varphi}_{i}}{\partial \overline{\mathbf{t}}_{k}}
\end{aligned}
$$

Indeed, $\frac{\partial \varphi_{i}}{\partial \mathbf{t}_{k}}$ depends on the choice of the tensor distance, and therefore a closedform expression for the mean tensor cannot be obtained in general. The mean tensor is obtained instead by means of a gradient-ascent evolution process:

$$
\begin{aligned}
\overline{\mathbf{t}}_{k}^{(t+1)} & =\overline{\mathbf{t}}_{k}^{(t)}+\Delta t \frac{\partial}{\partial \overline{\mathbf{t}}_{k}^{(t)}} \log (\mathcal{L}) \\
& =\overline{\mathbf{t}}_{k}^{(t)}+\Delta t \sum_{i=1}^{N} \frac{\alpha_{k} g_{k}\left(\mathbf{t}_{i} \mid \boldsymbol{\Theta}_{k}\right)}{\sum_{k=1}^{K} \alpha_{k} g_{k}\left(\mathbf{t}_{i} \mid \boldsymbol{\Theta}_{k}\right)}\left(\boldsymbol{\varphi}_{i}\left(\overline{\mathbf{t}}_{k}^{(t)}\right)\right)^{T} \boldsymbol{\Lambda}^{-1} \frac{\partial \boldsymbol{\varphi}_{i}\left(\overline{\mathbf{t}}_{k}^{(t)}\right)}{\partial \overline{\mathbf{t}}_{k}^{(t)}}
\end{aligned}
$$

If a random initialization is employed for the $\overline{\mathbf{t}}_{k}^{(0)}$, the iterative process is likely to get stuck in local maxima. Therefore, a k-means algorithm is performed previously to the gradient ascent, so as to obtain the initial mean tensors of each component of the mixture.

With regard to the estimation of the parameters $\boldsymbol{\Lambda}_{k}$ and $\alpha_{k}$, their derivation does not change with respect to a vector-valued MoG:

$$
\begin{gathered}
\hat{\boldsymbol{\Lambda}}_{k}=\frac{\sum_{i=1}^{N} p(k \mid i)\left(\boldsymbol{\varphi}_{i}\left(\overline{\mathbf{T}}_{k}\right)\right)\left(\boldsymbol{\varphi}_{i}\left(\overline{\mathbf{T}}_{k}\right)\right)^{T}}{\sum_{i=1}^{N} p(k \mid i)} \quad \hat{\alpha}_{k}=\frac{1}{N} \sum_{i=1}^{N} p(k \mid i) \\
p(k \mid i)=\frac{\alpha_{k} g_{k}\left(\mathbf{T}_{i} \mid \boldsymbol{\Theta}_{k}\right)}{\sum_{k=1}^{K} \alpha_{k} g_{k}\left(\mathbf{T}_{i} \mid \boldsymbol{\Theta}_{k}\right)}
\end{gathered}
$$

For the estimation of the complexity of the MoG, we will follow the approach by Figueiredo et al. 16, based on the use of the MDL (Minimum Description Length) criterion and implemented by means of a modified EM algorithm that leads to an integrated model selection and estimation procedure. A maximum number of 5 components was set for each mixture to run the complexity selection algorithm. In practice, for most of the segmentation experiments 3 or 4 components were obtained. It was additionally verified that if the above mentioned maximum was increased (say 6,7), more than 5 components were never selected. 


\subsection{Level Set Evolution}

Based on the well-known GAR model [15, the segmentation we seek to perform is formulated as the minimization of the following energy functional:

$$
E\left(\mathcal{C}, \boldsymbol{\Theta}_{\mathbf{1}}, \boldsymbol{\Theta}_{\mathbf{2}}\right)=-\int_{\Omega_{1}} \log p\left(\mathbf{T}(\mathbf{x}) \mid \boldsymbol{\Theta}_{\mathbf{1}}\right) d \mathbf{x}-\int_{\Omega_{2}} \log p\left(\mathbf{T}(\mathbf{x}) \mid \boldsymbol{\Theta}_{\mathbf{2}}\right) d \mathbf{x}+\nu|\mathcal{C}|
$$

where $p\left(\mathbf{T}(\mathbf{x}) \mid \boldsymbol{\Theta}_{\mathbf{i}}\right)$ follows the MoG model introduced in the preceding section. In order to perform the segmentation, the energy functional must be minimized with respect to the statistical parameters $\Theta_{\mathbf{i}}$ and to the segmenting surface, that is represented by means of the level set function $\phi$. This is done following the twostep Expectation-Maximization technique [17. For a fixed level set, the statistical parameters are updated with their ML estimators. Next, the segmenting surface is evolved following the level set equation

$$
\frac{\partial \phi}{\partial t}(\mathbf{x})=\delta(\phi)\left[\nu \nabla \cdot\left(\frac{\nabla \phi}{|\nabla \phi|}\right)-\frac{p\left(\mathbf{T}(\mathbf{x}) \mid \Theta_{1}\right)}{p\left(\mathbf{T}(\mathbf{x}) \mid \Theta_{2}\right)}\right]
$$

where $\delta(\phi)$ is the Dirac function. In the next section, we evaluate the performance of the method proposed through the experimentation on real DT-MRI data.

\section{Experimental Results and Comparisons}

Experiments were conducted on the extraction of the corpus callosum from a set of real DT-MRI data in order to validate the segmentation method proposed. Comparisons were also performed with a related recent approach in the literature.

Data acquisition: The employed dataset consists of DT-MRI volumes of 32 subjects, which were acquired on a 1.5 Tesla scanner. The acquisition parameters were: $b$ value $=1000 \mathrm{sec} / \mathrm{mm}^{2}, \mathrm{TE}=1000 \mathrm{msec}, \mathrm{TR}=89 \mathrm{msec}$, along six diffusionsensitizing directions. The images were obtained on 79 planes with $128 \times 128$ pixels per slice 1. An example of the resulting DT-MRI volumes is shown in Figure 1 where color coding of the main tensor orientation has been employed.

Experiments: As for initial contours, the body of the corpus callosum was roughly delineated for 3 central sagittal slices of the volume, upon the visualization of the fractional anisotropy, FA [18. The delineation and the resulting initial surface are shown for a sample volume in Figure 1.

In order to illustrate the overall performance for all the volumes in the employed dataset, in Figure 2 we reproduce all the obtained segmentation results. A successful segmentation was achieved for all cases, proving MoG to be a robust

\footnotetext{
${ }^{1}$ This volume dataset was kindly made available by the Signal Processing Institute at the École Polytechnique Fédérale de Laussane, within a research collaboration under the scope of the 6th Framework Program Network of Excellence SIMILAR. This is thankfully acknowledged.
} 

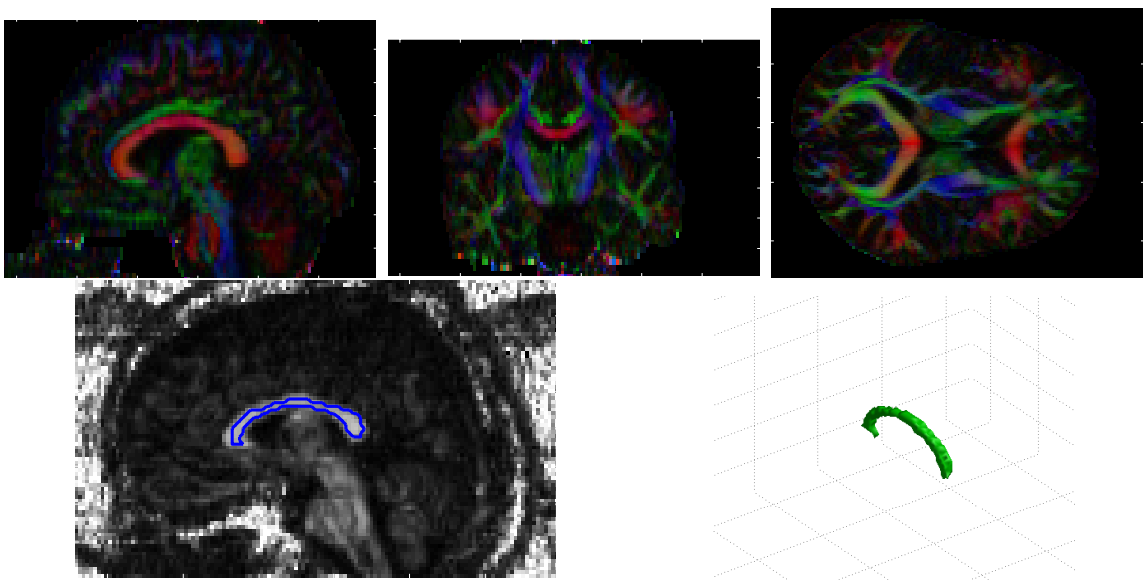

Fig. 1. Color coding of the main orientation in a sagittal, coronal and axial slice in a sample DT-MRI volume (top). Red means antero-posterior, green means left-right and blue inferior-superior. Delineation of the initial contour for the mid-sagittal slice, and initial level set surface (bottom).
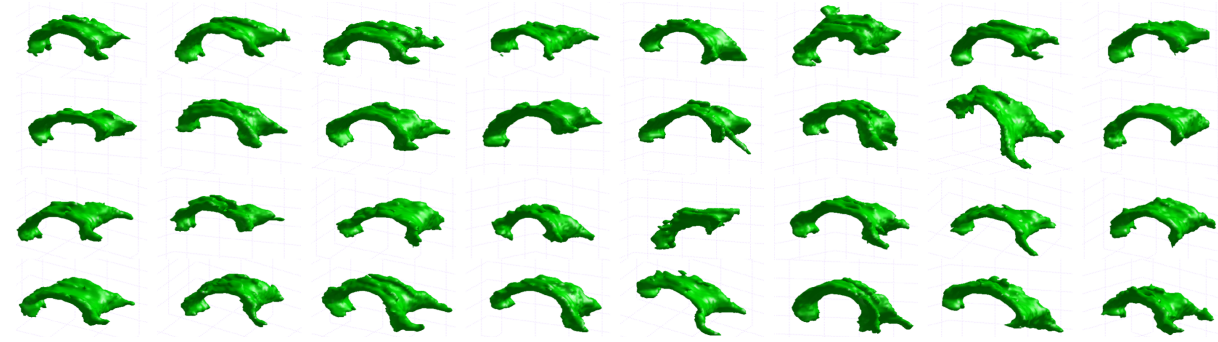

Fig. 2. Segmentation results for the corpus callosum of the 32 DT-MRI volumes using MoG on tensors proposed in this paper

model for the data. It can be also seen that the obtained segmentation is capable to capture both the genu and the splenium of the corpus callosum, which are the most difficult regions to segment.

For comparison purposes, we also show some segmentation results of our approach compared to those of the segmentation method in 1410, based on the Gaussian modeling of the tensor probability density function (geodesic distance was also employed for this segmentation method, together with the same initial surfaces and identical segmentation parameters). Although this approach was able to obtain good segmentation results on the employed dataset, it encounters some problems in the region of the splenium in some cases when compared to the MoG approach, and it shows lower accuracy for some other subjects. In Figure 3 , the segmentation results on a number of volumes show a better segmentation in the region of the splenium for the MoG model proposed in this paper. This 

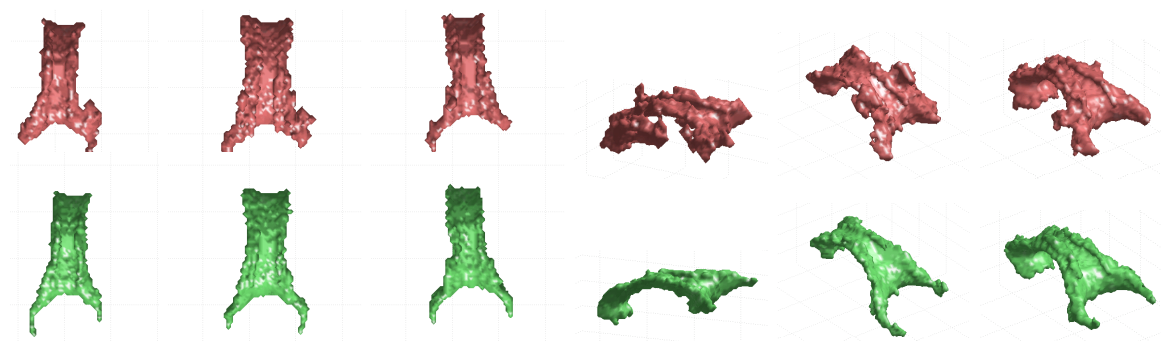

Fig. 3. Views of the segmentation results for the corpus callosum of different volumes using Gaussian model 14 10 (red) and MoG model proposed in this paper (green). Results show a better accuracy of our approach in the region of the splenium (three left cases), and a higher robustness to artifacts (three right cases).

increased performance is related to the ability of the MoG to capture a higher complexity in the data with respect to the single Gaussian model. In the same figure, some other segmentation results are shown where the MoG model shows a better accuracy than that of the Gaussian model, whose results present certain artifacts.

\section{Summary}

We have presented a novel technique for the segmentation of tensor fields applied to the extraction of anatomical structures from the brain white matter. The method is based on the definition of Gaussian mixtures on tensor-valued data, and experimental work on the segmentation of the corpus callosum from a large dataset of DT-MRI volumes shows a very good behaviour of the proposed model. Comparison with a recent and related approach based on a single Gaussian modeling is favorable in terms of the segmentation detail in specific areas such as the region of the splenium or robustness in the segmentation. We are currently working on the segmentation of other anatomical structures in the white matter and the shape analysis of the obtained structures.

\section{Acknowledgments}

The authors acknowledge the CICyT for research grant TEC2004-06647-C0301, the FIS for grant PI-041483 and the European Commission for the funds associated to the NoE SIMILAR (FP6- 507609).

\section{References}

1. Bihan, D.L., Breton, E., Lallemand, D., Grenier, P., Cabanis, E., Laval-Jeantet, M.: MR imaging of intravoxel incoherent motions: Application to diffusion and perfusion in neurologic disorders. Radiology 161, 401-407 (1986) 
2. Sundgren, P.C., Dong, Q., Gómez-Hassan, D., Mukherji, S.K., Maly, P., Welsh, R.: Diffusion tensor imaging of the brain: review of clinical applications. Neuroradiology 46, 339-350 (2004)

3. Horsfield, M., Jones, D.: Applications of diffusion-weighted and diffusion tensor MRI to white matter diseases-a review. NMR in Biomedicine 15, 570-577 (2002)

4. Zhukov, L., Museth, K., Breen, D., Whitaker, R., Barr, A.H.: Level set segmentation and modeling of DT-MRI human brain data. Journal of Electronic Imaging 12, 125-133 (2003)

5. Feddern, C., Weickert, J., Burgeth, B.: Level set methods for tensor-valued images. In: Proc. of the 9th IEEE Workshop on Variational, Geometric and Level Set Methods in Computer Vision, Nice, France, pp. 65-72 (2003)

6. Wiegell, M.R., Tuch, D.S., Larsson, H.B.W., Wedeen, V.J.: Automatic segmentation of thalamic nuclei from diffusion tensor magnetic resonance imaging. NeuroImage 19, 391-401 (2003)

7. Wang, Z., Vemuri, B.: An affine invariant tensor dissimilarity measure and its applications to tensor-valued image segmentation. In: Proc. of the IEEE Conference on CVPR, Washington DC, USA, pp. 228-233 (2004)

8. Lenglet, C., Rousson, M., Deriche, R.: Segmentation of 3d probability density fields by surface evolution: Application to diffusion MRI. In: Barillot, C., Haynor, D.R., Hellier, P. (eds.) MICCAI 2004. LNCS, vol. 3216, Springer, Heidelberg (2004)

9. Lenglet, C., Rousson, M., Deriche, R., Faugeras, O., Lehéricy, S., Ugurbil, K.: A Riemannian approach to diffusion tensor images segmentation. In: Proc. of Information Processing in Medical Imaging, Glenwood Springs, CO, USA (2005)

10. Lenglet, C., Rousson, M., Deriche, R.: DTI segmentation by statistical surface evolution. IEEE Transactions on Medical Imaging 25, 685-700 (2006)

11. Jonasson, L., Bresson, X., Hagmann, P., Cuisenaire, O., Meuli, R., Thiran, J.P.: White matter fiber tract segmentation in DT-MRI using geometric flows. Medical Image Analysis 9, 223-236 (2005)

12. Wang, Z., Vemuri, B.: Tensor field segmentation using region based active contour model. In: Proc. of the ECCV, Prague, Czech Republic, pp. 304-315 (2004)

13. Chan, T.F., Vese, L.A.: Active contours without edges. IEEE Trans. on Image Processing 10, 266-277 (2001)

14. Lenglet, C., Rousson, M., Deriche, R., Faugeras, O.: Statistics on the manifold of multivariate normal distributions: theory and application to diffusion tensor MRI processing. Journal of Mathematical Imaging and Vision 25, 423-444 (2006)

15. Paragios, N., Deriche, R.: Geodesic active regions: A new framework to deal with frame partition problems in computer vision. Journal of Visual Communication and Image Representation 13, 249-268 (2002)

16. Figueiredo, M., Leitao, J.M.N., Jain, A.K.: Unsupervised selection and estimation of finite mixture models. In: Proc. ICPR, Barcelona, Spain pp. 2087-2090 (2000)

17. Dempster, A., Laird, N., Rubin, D.: Maximum likelihood from incomplete data via the EM algorithm. Journal of the Royal Statistical Society 39, 1-38 (1977)

18. Basser, P., Pierpaoli, C.: Microstructural and physiological features of tissues elucidated by quantitative-diffusion tensor MRI. Journal of Magnetic Resonance B, 209-219 (1996) 